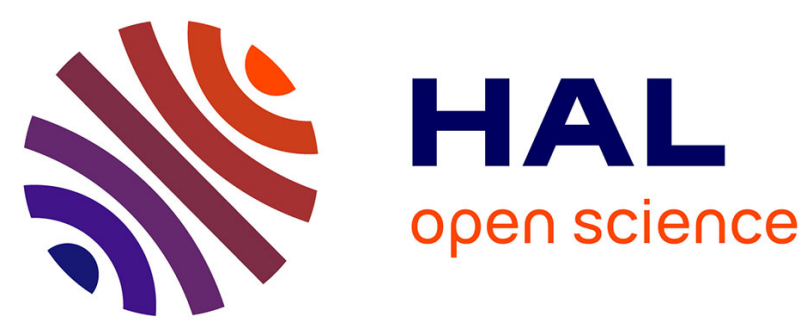

\title{
Distributed Consensus-formation of Force-controlled Nonholonomic Robots with Time-varying Delays
}

Emmanuel Nuno, Antonio Loria, Tonatiuh Henández, Mohamed Maghenem, Elena Panteley

\section{- To cite this version:}

Emmanuel Nuno, Antonio Loria, Tonatiuh Henández, Mohamed Maghenem, Elena Panteley. Distributed Consensus-formation of Force-controlled Nonholonomic Robots with Time-varying Delays. Automatica, 2020, 120, pp.109114. 10.1016/j.automatica.2020.109114 . hal-02901297

\section{HAL Id: hal-02901297 \\ https://hal.science/hal-02901297}

Submitted on 17 Jul 2020

HAL is a multi-disciplinary open access archive for the deposit and dissemination of scientific research documents, whether they are published or not. The documents may come from teaching and research institutions in France or abroad, or from public or private research centers.
L'archive ouverte pluridisciplinaire HAL, est destinée au dépôt et à la diffusion de documents scientifiques de niveau recherche, publiés ou non, émanant des établissements d'enseignement et de recherche français ou étrangers, des laboratoires publics ou privés. 


\title{
Distributed Consensus-formation of Force-controlled Nonholonomic Robots with Time-varying Delays
}

\author{
Emmanuel Nuño ${ }^{\mathrm{a}}$, Antonio Loría ${ }^{\mathrm{c}}$, Tonatiuh Henández ${ }^{\mathrm{a}}$, Mohamed Maghenem ${ }^{\mathrm{b}}$, \\ Elena Panteley c,d \\ ${ }^{a}$ Department of Computer Sciences, CUCEI, University of Guadalajara. Guadalajara, Jalisco, Mexico. \\ ${ }^{\mathrm{b}}$ Department of Computer Science and Engineering, University of California at Santa Cruz. California, USA. \\ ${ }^{\mathrm{c}}$ L2S-CentraleSupelec, CNRS, 3 Rue Joliot Curie, Gif-sur-Yvette, France. \\ ${ }^{\mathrm{d}}$ ITMO University, Kronverkskiy av. 49, Saint Petersburg, 197101, Russia.
}

\begin{abstract}
We solve the leaderless consensus-formation control problem for nonholonomic robots under the influence of time-varying communication delays and via smooth feedback. The control objective is to ensure that both the Cartesian positions of the vehicles and their orientations on the plane converge to a given position in a formation pattern, relatively to an $a$ priori unknown barycenter and a common orientation, respectively. Our controller, being of proportional-plus-damping type and smooth timevarying, is easy to implement. Furthermore, it relies on $\delta$-persistency of excitation to overcome the well-known obstacle that for nonholonomic systems a set-point is not stabilizable by smooth time-invariant feedback. We establish asymptotic convergence of the tracking errors and we provide some simulation results that support our theoretical findings.
\end{abstract}

Key words: Nonholonomic vehicles; formation control; consensus control; systems with time-delays.

\section{Introduction}

For multiagent systems the well-studied consensus control problem consists in making all the systems' states converge to a common equilibrium (Ren and Beard, 2005; Jadbabaie et al., 2003; Olfati-Saber and Murray, 2004). For mobile robots, because several of these can obviously not occupy the same position simultaneously, consensus must be formulated as a problem of formation control, that is, in which the robots stabilize near an equilibrium point while forming a geometric pattern (Lin et al., 2005). We may distinguish two consensus-formation problems: the leader-follower, in which the agreement equilibrium point is given as a desired value to a set of follower agents in the network (Maghenem, 2017), and the leaderless, in which such common value results from the network's topology (Cao and Ren, 2011; Wang, 2014; Hatanaka et al., 2015; Dong and Farrell, 2008a). Furthermore, it is important to distinguish works in which both Cartesian coordinates and orientation are controlled (Dimarogonas and Kyriakopoulos, 2007; Maghenem, 2017) from those in which orientation is neglected (Lin et al., 2005; Roza et al., 2018). In this paper we focus on the leaderless fullconsensus problem, that is, the set-point is not given a priori and consensus is reached both in Cartesian positions and orientation angles.

Email address: emmanuel.nuno@cucei.udg.mx (Emmanuel Nuño).
Another important distinction lies in the model considered. It can be of first order, in which case either only the kinematics resulting from the nonholonomic constraints is considered (Lin et al., 2005; Roza et al., 2018; Peng et al., 2015; Dong and Farrell, 2008a) or it may be simplified even further to a point-mass on the plane, in which case the consensus problem boils down to that of consensus of simple integrators; a problem completely solved under various scenarios (Ren and Beard, 2005; Ren, 2008; Olfati-Saber and Murray, 2004). A more realistic model, however, is force-controlled; this model is of second order and, besides the nonholonomy and underactuation, it presents the difficulty of controlling a higher relativedegree system. This is the model that we consider in this paper; see also (Do et al., 2004; Bautista-Castillo et al., 2016; Maghenem et al., 2019).

From a control viewpoint, the nonholonomy of the robot imposes significant difficulty to set-point stabilization; it is well known that these systems are not stabilizable via time-invariant smooth feedback (Brockett, 1983); for multiagent systems, necessary conditions for consensus are laid in (Lin et al., 2005). Thus, consensus of multiagent nonholonomic systems is solvable either via discontinuous time-invariant control (Dimarogonas and Kyriakopoulos, 2007) or time-varying smooth feedback (Yang et al., 2016; Jin and Gans, 2017).

From an implementation viewpoint, there are many kinds of controllers that may be designed depending 
on the available sensors and their capabilities. In this article we assume that the robots are equipped with global-measurement sensors and they transmit and receive measurements from "neighbor" robots that are interconnected over a network. It is most sensible to assume that communication flows in both directions (hence the network topology is undirected) and delays are time-varying (Hatanaka et al., 2015; Abdessameud et al., 2017). Such scenario is common, for instance, in the case that a group of robots are initially too far apart for relative-measurement sensors to be of any use. When within range, however, relative measurements may result more practical than global ones in missions of formation-tracking control (after the rendez-vous has been achieved). Relative-measurement based control is considered, for instance, in (Roza et al., 2018), but without communication delays and neglecting to control the orientation. Delays in the communication have been considered, e.g., in (Dong and Farrell, 2008b; Maghenem, 2017). In the former delays are considered to be constant and in the latter they are time-varying, but the control gains, as in (Roza et al., 2018), must satisfy a condition that requires global connectivity information.

Thus, in this paper we consider the full-consensus problem for second-order (force-controlled) nonholonomic systems interconnected over an undirected graph and with time-varying measurement delays. We propose a smooth controller of proportional-plus-damping type that achieves the control goal via a $\delta$-persistentlyexciting $(\delta-\mathrm{PE})$ term - $c f$. (Loría et al., 1999). Our controller is completely distributed as the control gains depend only on the actual strength of the interconnection for each agent. To the best of our knowledge, this problem, under the conditions considered here, has only been considered in (Maghenem, 2017). Other important aspects, such as collision avoidance (Jin and Gans, 2017) and robustness with respect to external disturbances (Ajorlou et al., 2015; Maghenem et al., 2018) are out of scope in this work.

The rest of the paper is organized as follows. In the next section we present the model and formulate the control problems that we address. In Section 3 we present our main theoretical results; these are illustrated via numerical simulations in Section 4. We conclude with some remarks in Section 5.

\section{Model and problem formulation}

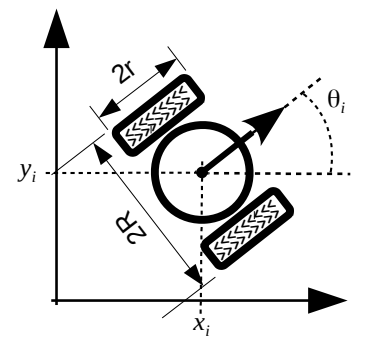

Fig. 1. Schematics of a differential wheeled mobile robot.
Consider a group of $N$ force-controlled nonholonomic vehicles, like the one depicted in Fig. 1, each of which is modeled by the usual unicycle kinematics equations and two added integrators, that is, for each $i \leq N$, we have

$\dot{z}_{i}=\varphi_{i}\left(\theta_{i}\right) v_{i}, \quad \varphi_{i}\left(\theta_{i}\right):=\left[\begin{array}{ll}\cos \left(\theta_{i}\right) & \sin \left(\theta_{i}\right)\end{array}\right]^{\top}$

$\dot{v}_{i}=u_{v i}$,

$\dot{\theta}_{i}=\omega_{i}$

$\dot{\theta}_{i}=\omega_{i}$

$\dot{\omega}_{i}=u_{\omega i}$,

$$
u_{\omega i}:=\frac{2 R_{i}}{I_{i} r_{i}}\left[\tau_{i 1}-\tau_{i 2}\right]
$$

where $z_{i}:=\left[x_{i}, y_{i}\right]^{\top} \in \mathbb{R}^{2}$ are the Cartesian coordinates of the robot on the plane, $\theta_{i} \in \mathbb{R}$ denotes its orientation, $v_{i}$ and $\omega_{i}$ are the linear and angular velocities of the center of mass, respectively, $m_{i}$ is the mass, $I_{i}$ is the moment of inertia, $r_{i}$ the radius of the wheels, $R_{i}$ is the distance between point $Q_{i}$ and the wheels, $u_{v i}$ and $u_{\omega i}$ are the control inputs, and $\tau_{i j}$ are the torques at the wheels, that is, for the purpose of control implementation, we have

$$
\left[\begin{array}{c}
\tau_{i 1} \\
\tau_{i 2}
\end{array}\right]=\frac{r_{i}}{2}\left[\begin{array}{cc}
m_{i} & I_{i} / 2 R_{i} \\
m_{i} & -I_{i} / 2 R_{i}
\end{array}\right]\left[\begin{array}{l}
u_{v i} \\
u_{\omega_{i}}
\end{array}\right] .
$$

The leaderless consensus-formation control problem, which we solve in this paper, involves making the robots acquire a desired formation pattern relatively to a barycenter with coordinates $z_{c}:=\left[\begin{array}{ll}x_{c} & y_{c}\end{array}\right]^{\top}$ and a common orientation, denoted $\theta_{c}$. It is assumed that both, $z_{c}$ and $\theta_{c}$ are unknown; they result from the topology of the interconnection graph and the multiagent system's initial conditions. The formation is determined by defining, for each robot, a constant vector denoted $\delta_{i} \in \mathbb{R}^{2}, \delta_{i}:=\left[\begin{array}{ll}\delta_{x i} & \delta_{y i}\end{array}\right]$ and the relative position errors $\bar{z}_{i}:=z_{i}-\delta_{i}$. Then, the control goal is to steer $\bar{z}_{i} \rightarrow z_{c}$ and $\theta_{i} \rightarrow \theta_{c}$ for all $i \leq N$.

We assume that each robot possesses position and velocity sensors and is able to communicate them to a number of neighbors through a relatively reliable network. More precisely, we assume that

(A1) the interconnection graph is undirected, static, and connected.

Hence, the interconnection graph may be modeled by a constant Laplacian matrix, $L:=\left[\ell_{i j}\right] \in \mathbb{R}^{N \times N}$, where

$$
\ell_{i j}=\left\{\begin{array}{cc}
\sum_{k \in \mathcal{N}_{i}} a_{i k} & i=k \\
-a_{i k} & i \neq k
\end{array}\right.
$$

$\mathcal{N}_{i} \subset \mathbb{Z}$ is the set of indexes corresponding to robots transmitting information to the $i$ th robot, $a_{i k}>0$ if $k \in \mathcal{N}_{i}$ and $a_{i k}=0$ otherwise. By construction, $L \mathbf{1}_{N}=$ 0 , where $\mathbf{1}_{N}=[1 \cdots 1]^{\top}$ and, after Assumption A1, $L$ is symmetric, it has a unique zero-eigenvalue, and the rest of its spectrum is strictly positive. Thus, $\operatorname{rank}(L)=$ $N-1$.

On the other hand, although the network interconnections are assumed to be static, the network is logically as- 
sumed to be wireless. This has as a natural consequence that the communications are affected by time-varying delays (Hatanaka et al., 2015; Abdessameud et al., 2017). Hence, we also assume that

(A2) the communication from the $j$ th to the $i$ th robot is subject to a variable time-delay denoted $T_{j i}(t)$ that is bounded by a known upper-bound $\bar{T}_{j i} \geq 0$ and has bounded time-derivatives.

Then, the control problem consists in designing $u_{v i}$ and $u_{\omega_{i}}$ so that

$$
\begin{aligned}
& \lim _{t \rightarrow \infty} v_{i}(t)=0, \quad \lim _{t \rightarrow \infty} \omega_{i}(t)=0, \\
& \lim _{t \rightarrow \infty} \bar{z}_{i}(t)=z_{c}, \quad \lim _{t \rightarrow \infty} \theta_{i}(t)=\theta_{c},
\end{aligned}
$$

for all $i \leq N$.

Remark 1 Being inherently a set-point stabilization problem, leaderless consensus differs greatly from other formation control problems, notably from moving-leaderfollower consensus, which is intrinsically a tracking control problem. Firstly, in clear contrast with the case of holonomic systems, set-point stabilization cannot be considered as a particular case of tractory tracking (Lizárraga., 2004). Secondly, from a systems theory viewpoint, an important distinction lies in that nonholonomic systems are controllable about a trajectory provided that the leader velocity is nonzero (de Luca et al., 2016). While this is relevant in tracking-control scenarii, in the present setting the main difficulty resides in that a set-point is not asymptotically stabilizable by smooth feedback, unless the latter is time-varying.

\section{Main result}

The main contribution in this paper is to provide an original solution to the problem previously stated: full leaderless consensus control of multiple second-order nonholonomic systems.

For the purpose of control design and analysis, we start by reformulating the control goal in (6) as

$$
\lim _{t \rightarrow \infty} e_{z i}(t)=0, \quad \lim _{t \rightarrow \infty} e_{\theta_{i}}(t)=0
$$

for all $i \leq N$, where

$$
e_{z i}:=\sum_{j \in \mathcal{N}_{i}} a_{i j}\left[\bar{z}_{i}-\bar{z}_{j}\left(t-T_{j i}(t)\right)\right]
$$

denotes the consensus errors in terms of the Cartesian coordinates of the $i$ th robot and the measurements received from its $\mathcal{N}_{i}$ neighbours, with different time-varying delays, and

$$
e_{\theta i}:=\sum_{j \in \mathcal{N}_{i}} a_{i j}\left[\theta_{i}-\theta_{j}\left(t-T_{j i}(t)\right)\right]
$$

denotes the consensus error in terms of the orientation angles, also affected by time-varying delays.

The control approach is based on designing a controller for each part of the system's dynamics separately. One for the dynamics corresponding to the translational part, Eq. (1), and another one for the orientation-angles dynamics. Since the respective systems (1) and (2) are of second order it is natural to design controllers of proportional-derivative type - cf. (Ren and Beard, 2005). Nonetheless, the system being subject to nonholonomic constraints, a set-point is not stabilizable via time-invariant smooth feedback (Brockett, 1983). Furthermore, because it is also desired to reach consensus in the orientation angles, controllers tailored for pointmass models do not apply either. Thus, our controller is of proportional-derivative type and it is smooth timevarying.

First, for the translational dynamics of the $i$ th robot we introduce the nonlinear control law $-c f$. (Nuño et al., 2013),

$$
u_{v i}=-p_{v i} \varphi_{i}\left(\theta_{i}\right)^{\top} e_{z i}-d_{v i} v_{i},
$$

where the parameters $p_{v i}$ and $d_{v i}$ are, respectively, the so-called proportional and derivative gains and are positive constants by design. The derivative term is simply proportional to the forward velocities $v_{i} \in \mathbb{R}$ but, in view of the robot's kinematics, the proportional-feedback term, $-p_{v i} \varphi_{i}\left(\theta_{i}\right)^{\top} e_{z i}$, which depends on the consensus errors $e_{z i} \in \mathbb{R}^{2}$, involves the rank-deficient vector $\varphi_{i}\left(\theta_{i}\right)$. Some readers may see here an analogy between the proportional-feedback term and the Jacobian-transpose controller used for robot manipulators, in which the Jacobian is not required to have full rank to achieve regulation.

Then, the closed-loop equation, corresponding to the translational dynamics, is

$$
\Sigma_{v}:\left\{\begin{array}{l}
\dot{\bar{z}}_{i}=\varphi_{i}\left(\theta_{i}\right) v_{i} \\
\dot{v}_{i}=-d_{v i} v_{i}-p_{v i} \varphi_{i}\left(\theta_{i}\right)^{\top} e_{z i} .
\end{array}\right.
$$

In contrast to the case of an ordinary double integrator the system (11) has multiple equilibria, other than points including $e_{z i}=0$. These equilibria correspond to points in the set $\mathcal{E}_{v}:=\left\{\bar{z}_{i}, \theta_{i}, v_{i}, e_{z_{i}}: v_{i}=0 \wedge \varphi_{i}\left(\theta_{i}\right)^{\top} e_{z i}=\right.$ $0\}$. This, in addition to the fact that set-points are not stabilizable for nonholonomic systems, via smooth timeinvariant feedback, considerably increases the difficulty of the consensus problem, relatively to that for linear systems or even systems with holonomic constraints.

The so-needed external function of time is introduced through the second control law, at the orientation-angle dynamics. Let

$$
u_{\omega i}=-p_{\omega i} e_{\theta i}-d_{\omega i} \omega_{i}+\alpha_{i}\left(t, \theta_{i}, e_{z i}\right),
$$

where $p_{\omega i}$ and $d_{\omega i}$ are positive gains. Clearly, the first two terms on the right-hand side of (12) are also of the proportional-derivative type. The function $\alpha_{i}$, which introduces the needed time-varying terms, is defined as

$$
\alpha_{i}\left(t, \theta_{i}, e_{z i}\right):=k_{\alpha i} f_{i}(t) \varphi_{i}\left(\theta_{i}\right)^{\perp \top} e_{z i},
$$

where $k_{\alpha i}>0, \varphi_{i}\left(\theta_{i}\right)^{\perp}=\left[-\sin \left(\theta_{i}\right) \cos \left(\theta_{i}\right)\right]^{\top}$ is the annihilator of $\varphi_{i}$, i.e., $\varphi_{i}\left(\theta_{i}\right)^{\top} \varphi_{i}\left(\theta_{i}\right)^{\perp}=\varphi_{i}\left(\theta_{i}\right)^{\perp \top} \varphi_{i}\left(\theta_{i}\right)=0$, 
and $f_{i} \in \mathcal{C}^{2}\left(\mathbb{R}_{\geq 0}, \mathbb{R}\right)$ satisfies, by construction, $f_{i}, \dot{f}_{i}, \ddot{f}_{i} \in$ $\mathcal{L}_{\infty}, \lim _{t \rightarrow \infty} f_{i}(t) \neq 0$, and $\lim _{t \rightarrow \infty} \dot{f}_{i}(t) \neq 0$.

The stabilization mechanism of $u_{\omega i}$ may be explained by observing the closed-loop equations relative to the orientation-angle dynamics,

$$
\Sigma_{\omega}:\left\{\begin{array}{l}
\dot{\theta}_{i}=\omega_{i} \\
\dot{\omega}_{i}=-d_{\omega i} \omega_{i}-p_{\omega i} e_{\theta i}+\alpha_{i}\left(t, \theta_{i}, e_{z i}\right) .
\end{array}\right.
$$

With $\alpha_{i} \equiv 0$, these equations correspond to those of a stable second-order time-invariant system with equilibria in $\mathcal{E}_{\omega}:=\left\{\theta_{i}, \omega_{i}: e_{\theta_{i}}=0, \omega_{i}=0\right\}$. Hence, without the term $\alpha_{i}$ in the controller, complete system's trajectories may belong to $\mathcal{E}_{\omega} \cap \mathcal{E}_{v}$ which includes, but is not restricted to, the consensus manifold. On the other hand, because $\varphi_{i}\left(\theta_{i}\right)$ and $\varphi_{i}\left(\theta_{i}\right)^{\perp}$ are orthogonal, we have $\varphi_{i}\left(\theta_{i}\right)^{\perp \top} e_{z i} \neq 0$ for all points in $\mathcal{E}_{v} \backslash\left\{v_{i}=0, e_{z i}=0\right\}$. Now, in view of the assumptions imposed on $f_{i}$ in (13), the function $\alpha_{i}$ evaluated along complete trajectories belonging to $\mathcal{E}_{v}$ cannot vanish. Thus, $\alpha_{i}$ may be seen as a "controlled perturbation" that persistently impedes all the trajectories, except the trivial one, to remain in $\mathcal{E}_{\omega} \cap \mathcal{E}_{v}$.

Remark 2 The excitation property of $\alpha_{i}$ is reminiscent of $\delta$-persistency of excitation (Loría et al., 2005). See also (Loría et al., 1999) where the so-called $\delta$-persistently-exciting controllers were introduced for stabilization of nonholonomic systems.

Our main result emerges from the previous rationale.

Proposition 1 Consider the system (1)-(2), under Assumptions A1 and A2, in closed loop with (10), (12)-(13) and with $f_{i}$ as defined above. Then, the leaderless consensus control goal is achieved, that is (5) and (6) hold, provided that

$$
\begin{aligned}
& d_{v i}>\frac{1}{2} p_{v i} \sum_{j \in \mathcal{N}_{i}} a_{i j}\left(\beta_{i}+\frac{\bar{T}_{i j}^{2}}{\beta_{j}}\right) \\
& d_{\omega i}>\frac{1}{2} p_{\omega i} \sum_{j \in \mathcal{N}_{i}} a_{i j}\left(\varepsilon_{i}+\frac{\bar{T}_{i j}^{2}}{\varepsilon_{j}}\right)
\end{aligned}
$$

for some $\beta_{i}, \varepsilon_{i}>0$, for all $i \leq N$.

\section{Proof of Proposition 1}

The proof relies on Barbalat's lemma and standard signal-chasing arguments. We start by showing that $v_{i} \rightarrow 0$ and that the solutions of $\Sigma_{v}$ are bounded. To that end, consider the Lyapunov-Krasovskii functional

$$
\begin{aligned}
\mathcal{V}:= & \sum_{i \leq N}\left[\frac{1}{2 p_{v i}} v_{i}^{2}+\frac{1}{4} \sum_{j \in \mathcal{N}_{i}} a_{i j}\left|\bar{z}_{i}-\bar{z}_{j}\right|^{2}\right. \\
& \left.+\frac{1}{2 \beta_{i}} \sum_{j \in \mathcal{N}_{i}} a_{i j} \bar{T}_{j i} \int_{\bar{T}_{j i}}^{0} \int_{t+\sigma}^{t} v_{j}(\eta)^{2} d \eta d \sigma\right]
\end{aligned}
$$

whose total derivative, along the trajectories of (11), yields

$$
\begin{aligned}
\dot{\mathcal{V}}=-\sum_{i \leq N} & {\left[\frac{d_{v i}}{p_{v i}} v_{i}^{2}+\dot{\bar{z}}_{i}^{\top} \sum_{j \in \mathcal{N}_{i}} a_{i j}\left(\bar{z}_{i}-\bar{z}_{j}\left(t-T_{j i}(t)\right)\right)\right.} \\
& -\frac{1}{2} \sum_{j \in \mathcal{N}_{i}} a_{i j}\left(\dot{\bar{z}}_{i}-\dot{\bar{z}}_{j}\right)^{\top}\left(\bar{z}_{i}-\bar{z}_{j}\right) \\
& \left.-\frac{1}{2 \beta_{i}} \sum_{j \in \mathcal{N}_{i}} a_{i j}\left(\bar{T}_{j i}^{2} v_{j}^{2}-\bar{T}_{j i} \int_{t-\bar{T}_{j i}}^{t} v_{j}^{2}(\sigma) d \sigma\right)\right] .
\end{aligned}
$$

Then, we use

$$
\bar{z}_{j}-\bar{z}_{j}\left(t-T_{j i}(t)\right)=\int_{t-T_{j i}(t)}^{t} \dot{\bar{z}}_{j}(\sigma) d \sigma,
$$

and Lemma 6.1 from (Cao and Ren, 2011) to obtain, under Assumption A1,

$\frac{1}{2} \sum_{i \leq N} \sum_{j \in \mathcal{N}_{i}} a_{i j}\left(\dot{\bar{z}}_{i}-\dot{\bar{z}}_{j}\right)^{\top}\left(\bar{z}_{i}-\bar{z}_{j}\right)=\sum_{i \leq N} \sum_{j \in \mathcal{N}_{i}} a_{i j} \dot{\bar{z}}_{i}^{\top}\left(\bar{z}_{i}-\bar{z}_{j}\right)$.

Therefore,

$$
\begin{aligned}
\dot{\mathcal{V}}=- & \sum_{i \leq N}\left[\frac{d_{v i}}{p_{v i}} v_{i}^{2}+\sum_{j \in \mathcal{N}_{i}} a_{i j} \dot{\bar{z}}_{i}^{\top} \int_{t-T_{j i}(t)}^{t} \dot{\bar{z}}_{j}(\sigma) d \sigma\right. \\
& \left.-\frac{1}{2 \beta_{i}} \sum_{j \in \mathcal{N}_{i}} a_{i j}\left(\bar{T}_{j i}^{2} v_{j}^{2}-\bar{T}_{j i} \int_{t-\bar{T}_{j i}}^{t} v_{j}^{2}(\sigma) d \sigma\right)\right] .
\end{aligned}
$$

Next, we apply Young's and Cauchy-Schwarz' inequalities on the second right-hand-term to obtain

$$
\begin{aligned}
-\dot{\bar{z}}_{i}^{\top} \int_{t-T_{j i}}^{t} \dot{\bar{z}}_{j}(\sigma) d \sigma & \leq \frac{\beta_{i}}{2}\left|\dot{\bar{z}}_{i}\right|^{2}+\frac{1}{2 \beta_{i}}\left|\int_{t-T_{j i}}^{t} \dot{\bar{z}}_{j}(\sigma) d \sigma\right|^{2} \\
& \leq \frac{\beta_{i}}{2}\left|\dot{\bar{z}}_{i}\right|^{2}+\frac{\bar{T}_{j i}}{2 \beta_{i}} \int_{t-\bar{T}_{j i}}^{t}\left|\dot{\bar{z}}_{j}(\sigma)\right|^{2} d \sigma
\end{aligned}
$$

for any $\beta_{i}>0$. Now, since $\varphi_{i}^{\top} \varphi_{i}=1$, we have $\left|\dot{\bar{z}}_{i}\right|^{2}=v_{i}^{2}$ and, in turn,

$$
\dot{\mathcal{V}} \leq-\sum_{i \leq N}\left[\left(\frac{d_{v i}}{p_{v i}}-\frac{\beta_{i}}{2} l_{i i}\right) v_{i}^{2}-\sum_{j \in \mathcal{N}_{i}} a_{i j} \frac{\bar{T}_{j i}^{2}}{2 \beta_{i}} v_{j}^{2}\right]
$$

where $l_{i i}:=\sum_{j \in \mathcal{N}_{i}} a_{i j}$ is the $i$ th element in the diagonal of the Laplacian matrix. Now, following (Nuño et al., 2013) and defining $s\left(v_{i}^{2}\right):=\left[v_{1}^{2} \cdots v_{N}^{2}\right]^{\top}$ and

$$
\Psi=\left[\begin{array}{cccc}
\frac{d_{v 1}}{p_{v 1}}-\frac{\beta_{1}}{2} l_{11} & -\frac{\bar{T}_{21}^{2}}{2 \beta_{1}} a_{12} & \ldots & -\frac{\bar{T}_{N 1}^{2}}{2 \beta_{1}} a_{1 N} \\
-\frac{T_{12}^{2}}{2 \beta_{2}} a_{21} & \frac{d_{v 2}}{p_{v 2}}-\frac{\beta_{2}}{2} l_{22} & \ldots & -\frac{\bar{T}_{N 2}^{2}}{2 \beta_{2}} a_{2 N} \\
\vdots & \vdots & \ddots & \vdots \\
-\frac{\bar{T}_{1 N}^{2}}{2 \beta_{N}} a_{N 1} & -\frac{\bar{T}_{2 N}^{2}}{2 \beta_{N}} a_{N 2} & \ldots & \frac{d_{v N}}{p_{v N}}-\frac{\beta_{N}}{2} l_{N N}
\end{array}\right]
$$


we obtain $\dot{\mathcal{V}} \leq-1_{N}^{\top} \Psi s\left(v_{i}^{2}\right)$ or, equivalently,

$$
\dot{\mathcal{V}} \leq-\sum_{i \leq N}\left[\frac{d_{v i}}{p_{v i}}-\sum_{j \in \mathcal{N}_{i}} a_{i j}\left(\frac{\beta_{i}}{2}+\frac{\bar{T}_{i j}^{2}}{2 \beta_{j}}\right)\right] v_{i}^{2}
$$

Thus, after (15), it follows that there exists $\lambda_{i}>0$ such that $\dot{\mathcal{V}} \leq-\sum_{i<N} \lambda_{i} v_{i}^{2}$. This implies that $v_{i} \in \mathcal{L}_{2}$ and, since $\mathcal{V}$ is positive definite and radially unbounded with respect to $v_{i}$ and $\bar{z}_{i}-\bar{z}_{j}$, the latter belong to $\mathcal{L}_{\infty}$ and, consequently, $e_{z i} \in \mathcal{L}_{\infty}$. Now, since $\varphi_{i}$ is uniformly bounded, it follows from (11) that $\dot{v}_{i} \in \mathcal{L}_{\infty}$ and, therefore, $\lim _{t \rightarrow \infty} v_{i}(t)=0$.

So far, we have established the first limit in (5). Now, we analyze the behavior of the solutions of (14) with the aim at establishing the second limit. To that end, consider the system $\Sigma_{\omega}$ with (for the time-being) $\alpha_{i} \equiv 0$ and the Lyapunov-Krasovskii functional

$$
\begin{aligned}
\mathcal{W}:=\sum_{i \leq N}[ & \frac{1}{2 p_{\omega i}} \omega_{i}^{2}+\frac{1}{4} \sum_{j \in \mathcal{N}_{i}} a_{i j}\left(\theta_{i}-\theta_{j}\right)^{2} \\
& \left.+\frac{1}{2 \varepsilon_{i}} \sum_{j \in \mathcal{N}_{i}} a_{i j} \bar{T}_{j i} \int_{-\bar{T}_{j i}}^{0} \int_{t+\sigma}^{t} \omega_{j}^{2}(\eta) d \eta d \sigma\right],
\end{aligned}
$$

where $\varepsilon_{i}>0$. Proceeding as we did previously for the differentiation of $\mathcal{V}$, we obtain

$$
\dot{\mathcal{W}} \leq-\sum_{i \leq N}\left[\left(\frac{d_{\omega i}}{p_{\omega i}}-\frac{\varepsilon_{i}}{2} l_{i i}\right) \omega_{i}^{2}-\sum_{j \in \mathcal{N}_{i}} a_{i j} \frac{\bar{T}_{j i}^{2}}{2 \varepsilon_{i}} \omega_{j}^{2}\right]
$$

so

$$
\dot{\mathcal{W}} \leq-\sum_{i \leq N}\left(\frac{d_{\omega i}}{p_{\omega i}}-\sum_{j \in \mathcal{N}_{i}} a_{i j}\left(\frac{\varepsilon_{i}}{2}+\frac{\bar{T}_{i j}^{2}}{2 \varepsilon_{j}}\right)\right) \omega_{i}^{2} .
$$

Therefore, after (16), it follows that there exists $\lambda_{i}>0$ such that $\dot{\mathcal{W}} \leq-\sum_{i \leq N} \lambda_{i} \omega_{i}^{2}$. Since $\mathcal{W}$ is positive definite and radially unbounded with regards to $\omega_{i}$ and $\theta_{i}-\theta_{j}$, it follows that $\omega_{i} \in \mathcal{L}_{2} \cap \mathcal{L}_{\infty}$ and $\theta_{i}-\theta_{j} \in \mathcal{L}_{\infty}$. This, in turn, implies that $\dot{\omega}_{i} \in \mathcal{L}_{\infty}$ so, after Barbalat's Lemma, $\lim _{t \rightarrow \infty} \omega_{i}(t)=0$.

We proceed now to establish the limit in (6). To that end, observe that, since

$$
\lim _{t \rightarrow \infty} \int_{0}^{t} \dot{\omega}_{i}(\sigma) d \sigma=\lim _{t \rightarrow \infty} \omega_{i}(t)-\omega_{i}(0)=\omega_{i}(0),
$$

it follows, from Barbalat's lemma, that $\lim _{t \rightarrow \infty} \dot{\omega}_{i}(t)=0$ if $\dot{\omega}_{i}$ is uniformly continuous. The latter holds since

$$
\ddot{\omega}_{i}=-\frac{1}{I_{i}}\left[p_{\omega i} \dot{e}_{\theta i}+d_{\omega i} \dot{\omega}_{i}\right]
$$

is bounded; indeed, this follows from Assumption A2 and the fact that $\dot{\omega}_{i}, \omega_{i} \in \mathcal{L}_{\infty}$.

Now, from the second equation in (14), restricted to $\alpha_{i} \equiv$ 0 , it follows that $\lim _{t \rightarrow \infty} \dot{\omega}_{i}(t)=0$ and $\lim _{t \rightarrow \infty} \omega_{i}(t)=0$ imply that $\lim _{t \rightarrow \infty} e_{\theta i}(t)=0$ or, equivalently, that

$$
\lim _{t \rightarrow \infty} \sum_{j \in \mathcal{N}_{i}} a_{i j}\left(\theta_{i}(t)-\theta_{j}(t)+\int_{t-T_{j i}(t)}^{t} \omega_{j}(\sigma) d \sigma\right)=0 .
$$

Then, since $\omega_{i} \rightarrow 0$ and $T_{j i}(t)$ is globally bounded (see A2), we have

$$
\lim _{t \rightarrow \infty} \sum_{j \in \mathcal{N}_{i}} a_{i j}\left[\theta_{i}(t)-\theta_{j}(t)\right]=0
$$

which implies that $\lim _{t \rightarrow \infty} L \theta(t)=0$, where $\theta=\operatorname{col}\left(\theta_{i}\right) \in$ $\mathbb{R}^{N}$. Thus,

$$
\lim _{t \rightarrow \infty} \theta_{i}(t)=\theta_{c}
$$

All the previous arguments hold provided that $\alpha_{i} \equiv 0$. If this is not the case, we remark that since, $f_{i}, e_{z i}$ and $\varphi_{i}^{\perp}$ are bounded along trajectories, so is $\alpha_{i}-$ see Eq. (13). This and the fact that $\Sigma_{\omega}$ is a marginally stable linear time-varying system with uniformly bounded time-delays ensure that $\dot{\omega}_{i}, \omega_{i} \in \mathcal{L}_{\infty}$, by Proposition 3 in (Wang, 2020) and so is $e_{\theta i}$.

The rest of the proof mostly consists in establishing that $e_{z i} \rightarrow 0$ which, since $v_{i} \rightarrow 0$, implies that $\bar{z}_{i} \rightarrow \bar{z}_{c}$, thereby completing (6). To see the latter, note that

$$
e_{z i}=\sum_{j \in \mathcal{N}_{i}} a_{i j}\left(\bar{z}_{i}-\bar{z}_{j}+\int_{t-T_{j i}(t)}^{t} \dot{\bar{z}}_{j}(\sigma) d \sigma\right),
$$

so, defining $e_{z}:=\left[e_{z 1} \cdots e_{z N}\right]^{\top}$ and $\bar{z}:=\left[\bar{z}_{1} \cdots \bar{z}_{N}\right]$ respectively, and using $\dot{\bar{z}}_{j}(t)=\varphi_{j}(t) v_{j}(t)$, we obtain

$e_{z}=\left(L \otimes I_{2}\right) \bar{z}+\operatorname{col}\left(\sum_{j \in \mathcal{N}_{i}} a_{i j} \int_{t-T_{j i}(t)}^{t} \varphi_{j}(\sigma) v_{j}(\sigma) d \sigma\right)$.

Therefore, $e_{z i}=0$ and $v_{i}=0$ imply that $\left(L \otimes I_{2}\right) \bar{z}=0$ which, in view of the properties of $L$, implies the existence of $\bar{z}_{c} \in \mathbb{R}^{2}$ such that $\bar{z}=1_{N} \otimes \bar{z}_{c}$, or $\bar{z}_{i}(t)=\bar{z}_{c}$ for all $i \leq n$.

We proceed now to show that $e_{z i} \rightarrow 0$. To that end, we stress that, since $\lim _{t \rightarrow \infty} v_{i}(t)=0$,

$$
\lim _{t \rightarrow \infty} \int_{0}^{t} \dot{v}_{i}(\sigma) d \sigma=\lim _{t \rightarrow \infty} v_{i}(t)-v_{i}(0)=v_{i}(0) .
$$

Moreover, in view of Assumption A2 and the fact that $\theta_{i}, \omega_{i}, \dot{\omega}_{i}, v_{i}, \dot{v}_{i}$, and $e_{z i} \in \mathcal{L}_{\infty}$, so does

$$
\ddot{v}_{i}=-\frac{p_{v i}}{m_{i}}\left[\omega_{i} \varphi_{i}^{\perp \top} e_{z i}+\varphi_{i}^{\top} \dot{e}_{z i}+\frac{d_{v i}}{p_{v i}} \dot{v}_{i}\right] .
$$


After Barbalat's lemma, it follows that $\lim _{t \rightarrow \infty} \dot{v}_{i}(t)=0$ and, in turn after (11), also $\lim _{t \rightarrow \infty} \varphi_{i}(\theta(t))^{\top} e_{z i}(t)=0$. Note, however, that the solutions to $\varphi_{i}(\theta)^{\top} e_{z i}=0$ are of the form $e_{z i}=c \varphi_{i}^{\perp}$. Notwithstanding, so are the solutions to $\varphi_{i}(\theta)^{\perp \top} e_{z i}=0$ on the right-hand side of (18). Therefore, the unique solution to both equations is $c=0$ or, equivalently, $e_{z_{i}}=0$, since $\varphi_{i}^{\perp \top} \varphi_{i}^{\perp}=1$.

Thus, it is left to establish that $\varphi_{i}(\theta(t))^{\perp \top} e_{z i}(t) \rightarrow 0$. To that end, we use once more Barbalat's lemma to show that $\ddot{v}_{i} \rightarrow 0$. First, note that

$$
\lim _{t \rightarrow \infty} \int_{0}^{t} \ddot{v}_{i}(\sigma) d \sigma=\lim _{t \rightarrow \infty} \dot{v}_{i}(t)-\dot{v}_{i}(0)=-\dot{v}_{i}(0) .
$$

Then, to show that $\ddot{v}_{i}$ is uniformly continuous we use (18) and $\dot{\varphi}^{\perp}=-\omega_{i} \varphi_{i}$ to obtain

$$
\begin{gathered}
\dddot{v}_{i}=-\frac{p_{v i}}{m_{i}}\left[\dot{\omega}_{i} \varphi_{i}^{\perp \top} e_{z i}+2 \omega_{i} \varphi_{i}^{\perp \top} \dot{e}_{z i}-\omega_{i}^{2} \varphi_{i}^{\top} e_{z i}\right. \\
\left.+\varphi_{i}^{\top} \ddot{e}_{z i}+\frac{d_{v i}}{p_{v i}} \ddot{v}_{i}\right]
\end{gathered}
$$

which is bounded, in view of Assumption A2 and the boundedness of all functions on the right-hand side. This implies that $\lim _{t \rightarrow \infty} \ddot{v}_{i}(t)=0$. Similar arguments apply to establish that $\lim _{t \rightarrow \infty} \dddot{v}_{i}(t)=0$ and $\lim _{t \rightarrow \infty} v_{i}^{(4)}(t)=0$, where

$$
\begin{gathered}
v_{i}^{(4)}=-\frac{p_{v i}}{m_{i}}\left[\ddot{\omega}_{i} \varphi_{i}^{\perp \top} e_{z i}+3 \dot{\omega}_{i} \varphi_{i}^{\perp \top} \dot{e}_{z i}-\omega_{i}^{3} \varphi_{i}^{\perp \top} e_{z i}+\varphi_{i}^{\top} \dddot{e}_{z i}\right. \\
\left.+3 \omega_{i}\left(\varphi_{i}^{\perp \top} \ddot{e}_{z i}+\dot{\omega}_{i} \varphi_{i}^{\top} e_{z i}+\omega_{i} \varphi_{i}^{\top} \dot{e}_{z i}\right)+\frac{d_{v i}}{p_{v i}} \dddot{v}_{i}\right]
\end{gathered}
$$

Hence, since $v_{i}, \dot{v}_{i}, \ddot{v}_{i}, \dddot{v}_{i}, v_{i}^{(4)}$ and $\varphi_{i}^{\top} e_{z i}$ converge to zero, so do $\dot{e}_{z i}, \ddot{e}_{z i}$ and $\dddot{e}_{z i}$. It follows that all the terms in (18), (19), and (20) converge to zero. Thus, from (18) it follows that $\omega_{i} \varphi_{i}^{\perp \top} e_{z i} \rightarrow 0$, from (19) we have $\dot{\omega}_{i} \varphi_{i}^{\perp \top} e_{z i} \rightarrow 0$, and (20) implies that $\ddot{\omega}_{i} \varphi_{i}^{\perp \top} e_{z i} \rightarrow 0$.

Next, we use

$$
\begin{aligned}
\ddot{\omega}_{i}=-\frac{k_{\alpha i}}{I_{i}} & {\left[\frac{p_{\omega i}}{k_{\alpha i}} \dot{e}_{\theta i}+\frac{d_{\omega i}}{k_{\alpha i}} \dot{\omega}_{i}+f_{i} \omega_{i} \varphi_{i}^{\top} e_{z i}-f_{i} \varphi_{i}^{\perp \top} \dot{e}_{i}\right.} \\
& \left.-\dot{f}_{i} \varphi_{i}^{\perp \top} e_{z i}\right]
\end{aligned}
$$

in the first right hand side term of (20) to conclude that the term $\frac{p_{\omega i}}{k_{\alpha i}} \dot{e}_{\theta i} \varphi_{i}^{\perp \top} e_{z i}-\dot{f}_{i}\left(\varphi_{i}^{\perp \top} e_{z i}\right)^{2}$ converges to zero. Since, on one hand $\omega_{i} \varphi_{i}^{\perp \top} e_{z i}$ converges to zero and, on the other hand, $e_{z i}=c \varphi_{i}^{\perp}$ and $\varphi_{i}^{\perp \top} \varphi_{i}^{\perp}=1$, it follows that $\omega_{i}$ also converges to zero. In turn, we also have $\dot{e}_{\theta i} \varphi_{i}^{\perp \top} e_{z i} \rightarrow 0$. Finally, the design of $f_{i}$ guarantees that $\dot{f}_{i}$ does not vanish, so we conclude that $\lim _{t \rightarrow \infty} \varphi_{i}^{\perp \top}\left(\theta_{i}(t)\right) e_{z i}(t)=0$. Therefore $\alpha_{i}$ vanishes and the limits in (5), as well as (17), follow.

\section{Simulations}

We present now some numerical simulation results to illustrate our theoretical findings. The simulations are performed using a network of six differential-drive robots interconnected as illustrated in Fig. 2, below. For simplicity, the physical parameters of all robots are taken to be equal: $10 \mathrm{Kg}$ for the mass; $3 \mathrm{Kgm}^{2}$ for the moment of inertia; $0.3 \mathrm{~m}$ of the distance between point $Q$ and the wheels, and $0.05 \mathrm{~m}$ for the radius of the wheels $c f$. (Shojaei et al., 2011).

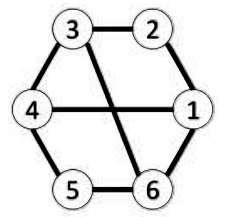

\begin{tabular}{c|cccccc|} 
Rовот & 1 & 2 & 3 & 4 & 5 & 6 \\
\hline$\delta_{x i}$ & 2 & 1 & -1 & -2 & -1 & 1 \\
$\delta_{y i}$ & 0 & 2 & 2 & 0 & -2 & -2 \\
\hline
\end{tabular}

Fig. 2. Communication topology and hexagonal desired formation pattern (left). The latter is defined as an hexagon, determined by the distances $\delta_{x i}$ and $\delta_{y i}$ as per in the table on the right.

The interconnection time-delays are defined as a normal Gaussian distribution with a mean of $0.2 \mathrm{~s}$ and a variance of $0.001 \mathrm{~s}$ hence, they are bounded by $\bar{T}_{j i}=0.33 \mathrm{~s}$. The functions $t \mapsto f_{i}$ are designed using a truncated Fourier series of a square wave, that is,

$$
f_{i}(t)=\frac{5}{2}+\sum_{k=1}^{5} \frac{4}{(2 k-1) \pi} \sin \left(\frac{2 k-1}{2} t\right) \quad \forall i \leq N .
$$

The robots are assumed to be initially standing still at the coordinates

$x_{i}(0):=\left[\begin{array}{llllll}5 & 7 & 7 & 3 & 1 & 1\end{array}\right]^{\top} y_{i}(0):=\left[\begin{array}{llllll}2 & 5.5 & 3.5 & 2 & 3.5 & 5.5\end{array}\right]^{\top}$

and with the following orientations (in rad)

$$
\theta(0)=\left[\begin{array}{llll}
\frac{\pi}{2} & 0 & -\frac{\pi}{8} & \frac{\pi}{8}-\frac{\pi}{8}-\frac{\pi}{8}
\end{array}\right]^{\top} .
$$

The controller gains are set to $p_{\omega i}=30, d_{\omega i}=60, k_{\alpha i}=$ $150, p_{v i}=300$ and $d_{v i}=600$, for all $i \leq N$.

As it may be appreciated from Fig. 4, the robots converge to the desired formation pattern with a barycenter at $z_{c}=[3.35,3.96]^{\top}$; their orientations converge to a common angle $\theta_{c}=5.77 \mathrm{rad}$, - see Fig. 3 below.

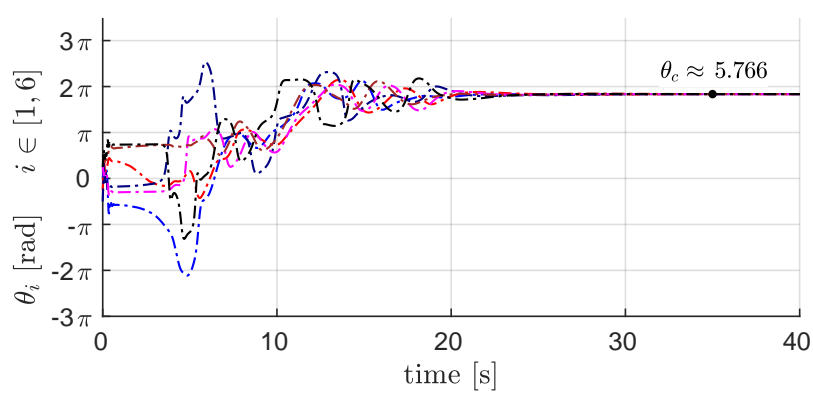

Fig. 3. Orientations of the six robots against time 


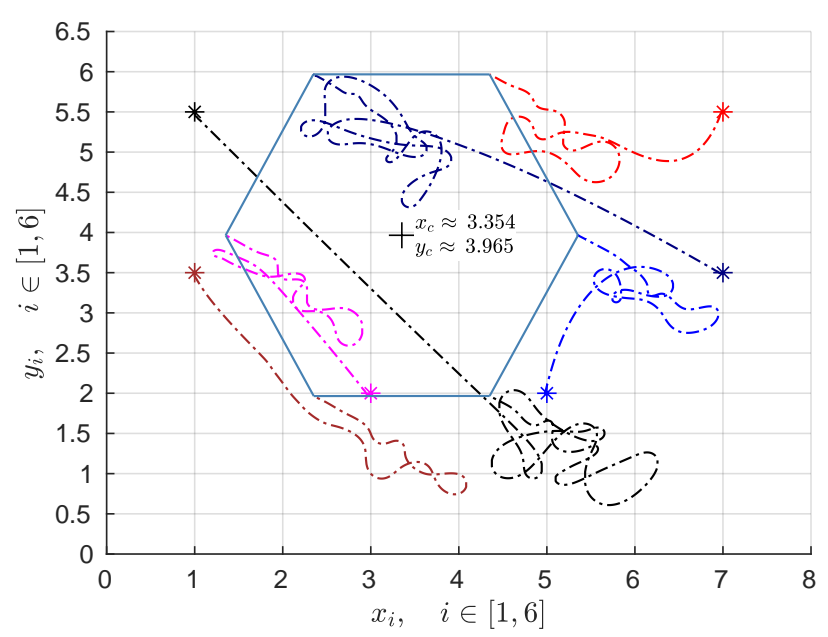

Fig. 4. Position trajectories in the Cartesian $x y$-plane

\section{Conclusions}

A solution to the leaderless consensus problem for force-controlled nonholonomic robots, in the presence of time-varying measurement delays, was presented. To cope with the difficulty imposed by the nonholonomic constraints to achieve set-point stabilization via smooth feedback, our controller is time-varying. It relies on a condition of persistency of excitation imposed on the term $\alpha_{i}\left(t, \theta_{i}, e_{z i}\right)$ - see Eq. (13). Such condition is reminiscent of $\delta$-persistency of excitation, which for nonlinear time-varying systems is necessary for uniform asymptotic stability (Loría et al., 2005). Even though this property is not established here (it is subject of current research), the previous rationale justifies the little conservativism of our assumptions.

From an implementation viewpoint, it is important to stress that the controllers have a simple proportionalplus-damping structure. Hence, the stability conditions (15) and (16) reflect the obvious fact that if the proportional gains are increased, to obtain a faster response, the dissipation gain must also be increased. Surprisingly, even though the system exhibits nonholonomic restrictions, the resulting conditions are the same as that reported in (Nuño et al., 2013) for the unrestricted case. On the other hand, the choice of $f_{i}$ also impacts the performance of the controller.

Current research focusses on moving-leader-follower consensus, on establishing stronger stability properties, and on determining a performance-driven tunning approach of the controller parameters.

\section{Acknowledgment}

This article is supported by the Mexican CONACyT Basic Scientific Research grant CB-282807, by the University of Paris Saclay, by the French ANR via project HANDY, number ANR-18-CE40-0010, by CEFIPRA under the grant number 6001-A, and by the Government of Russian Federation grant 074-U01.

\section{References}

A. Abdessameud, A. Tayebi, and I. G. Polushin. Leaderfollower synchronization of Euler-Lagrange systems with time-varying leader trajectory and constrained discretetime communication. IEEE Trans. on Automatic Control, 62(5):2539-2545, 2017.

A. Ajorlou, M.M. Asadi, M.G. Aghdam, and S. Blouin. Distributed consensus control of unicycle agents in the presence of external disturbances. Systems $\mathscr{E}$ Control Letters, 82:86-90, 2015.

A. Bautista-Castillo, C. Lopez-Franco, and E. Nuno. Consensus-based formation control for multiple nonholonomic robots. In IEEE International Autumn Meeting on Power, Electronics and Computing. IEEE, 2016.

R. W. Brockett. Asymptotic stability and feedback stabilization. Differential geometric control theory, 27(1):181-191, 1983.

Y. Cao and W. Ren. Distributed Coordination of Multiagent Networks: Emergent Problems, Models, and Issues. Springer-Verlag, 2011.

A. de Luca, G. Oriolo, and M. Vendittelli. Control of Wheeled Mobile Robots: An Experimental Overview, volume 270 of Lecture Notes in Control and Information Sciences, chapter in "Ramsete". Springer Verlag, Nicosia S., Siciliano B., Bicchi A., Valigi P. (eds), London, 2016.

D.V. Dimarogonas and K.J. Kyriakopoulos. On the rendezvous problem for multiple nonholonomic agents. IEEE Trans. on Automatic Control, 52(5):916-922, 2007.

K. D. Do, Z.-P. Jiang, and J. Pan. A global output-feedback controller for simultaneous tracking and stabilization of unicycle-type mobile robots. IEEE Trans. on Robotics Automat., 20(3):589-594, 2004.

A. Dong and J. A. Farrell. Cooperative control of multiple nonholonomic mobile agents. IEEE Trans. on Automatic Control, 53(6):1434-1447, 2008a.

W. Dong and J.A. Farrell. Consensus of multiple nonholonomic systems. In Decision and Control, 2008. CDC 2008. 47th IEEE Conference on, pages 2270-2275. IEEE, 2008b.

T. Hatanaka, N. Chopra, M. Fujita, and M.W. Spong. Passivity-Based Control and Estimation in Networked Robotics. Communications and Control Engineering. Springer, 2015.

P. Ioannou and J. Sun. Robust adaptive control. Prentice Hall, New Jersey, USA, 1996.

A. Jadbabaie, J. Lin, and A.S. Morse. Coordination of groups of mobile autonomous agents using nearest neighbor rules. IEEE Trans. on Automatic Control, 48(6):988-1001, 2003.

J. Jin and N. Gans. Collision-free formation and heading consensus of nonholonomic robots as a pose regulation problem. Robotics and Autonomous Systems, 95(9):25-36, 2017.

Z. Lin, B. Francis, and M. Maggiore. Necessary and sufficient graphical conditions for formation control of unicycles. IEEE Trans. on Automatic Control, 50(1):121-127, 2005.

D. A. Lizárraga. Obstructions to the existence of universal stabilizers for smooth control systems. Mathematics of Control, Signals and Systems, 16:255-277, 2004.

A. Loría, E. Panteley, and A. Teel. A new persistency-ofexcitation condition for UGAS of NLTV systems: Application to stabilization of nonholonomic systems. In Proc. 5th. European Contr. Conf., pages 1363-1368, Karlsrühe, Germany, 1999. 
A. Loría, E. Panteley, D. Popović, and A. Teel. A nested Matrosov theorem and persistency of excitation for uniform convergence in stable non-autonomous systems. IEEE Trans. on Automat. Contr., 50(2):183-198, 2005.

M. Maghenem. Stability and stabilization of networked varying systems. PhD thesis, Univ Paris Saclay, Gif sur Yvette, 2017.

M. Maghenem, A. Loría, and E. Panteley. A robust $\delta$ persistently exciting controller for leader-follower trackingagreement of multiple vehicles. European J. of Control, 40: $1-12,2018$

M. Maghenem, A. Bautista-Castillo, E. Nuño, A. Loría, and E. Panteley. Consensus of multi-agent systems with nonholonomic restrictions via Lyapunov's direct method. IEEE Control Systems Letters, 3(2):344-349, 2019.

E. Nuño, I. Sarras, and L. Basañez. Consensus in networks of nonidentical Euler-Lagrange systems using $\mathrm{P}+\mathrm{d}$ controllers. IEEE Trans. on Robotics, 26(6):1503-1508, 2013.

R. Olfati-Saber and R.M. Murray. Consensus problems in networks of agents with switching topology and timedelays. IEEE Trans. on Automatic Control, 49(9):15201533, 2004.

Z. Peng, G. Wen, A. Rahmani, and Y. Yu. Distributed consensus-based formation control for multiple nonholonomic mobile robots with a specified reference trajectory. International Journal of Systems Science, 46(8): 1447-1457, 2015

W. Ren. On consensus algorithms for double-integrator dynamics. IEEE Trans. on Automatic Control, 53(6):15031509, 2008.

W. Ren and R. W. Beard. Distributed consensus in multivehicle cooperative control. Springer Verlag, 2005.

A. Roza, M. Maggiore, and L. Scardovi. A smooth distributed feedback for global rendezvous of unicycles. IEEE Trans. on Control of Network Systems, 5(1):640-652, March 2018.

K. Shojaei, A.M. Shahri, A. Tarakameh, and B. Tabibian. Adaptive trajectory tracking control of a differential drive wheeled mobile robot. Robotica, 29(3):391-402, 2011.

H. Wang. Consensus of networked mechanical systems with communication delays: A unified framework. IEEE Trans. on Automatic Control, 59(6):1571-1576, 2014.

H. Wang. Differential-cascade framework for consensus of networked Lagrangian systems. Automatica, 112:108620, 2020 .

C. Yang, W. Xie, C. Lei, and B. Ma. Smooth time-varying formation control of multiple nonholonomic agents. In Proceedings of the 2015 Chinese Intelligent Systems Conference, pages 283-291. Springer, 2016.

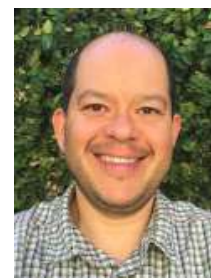

Emmanuel Nuño was born in Guadalajara Mexico, in 1980. He received the B.Sc. degree in communications and electronics engineering from the University of Guadalajara, Guadalajara, in 2002, and the Ph.D. degree in advanced automation and robotics from the Technical University of Catalonia Barcelona, Spain, in 2008. Since 2009, he has been a Titular Professor with the Department of Computer Science, University of Guadalajara. He is currently an Editor of the International Journal of Adaptive Control and Signal Processing. His research interests include the control of robots, of bilateral teleoperators and of networks of robots.

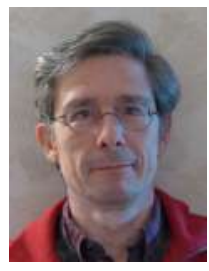

Antonio Loría obtained his BSc degree on Electronics Engineering from ITESM, Monterrey, Mexico in 1991 and his MSc and PhD degrees in Control Engg. from UTC, France in 1993 and 1996, respectively. A. Loría has the honour of being a tenure researcher at the French National Centre of Scientific Research (CNRS) since january 1999 (Senior Researcher since 2006). He has produced over 250 publications on control systems and stability theory and he has over 45 years of accumulated experience as associate and guest editor for major journals on automatic control. He was commended "Outstanding AE" by the EiCs of Automatica and IEEE Control Systems Letters.

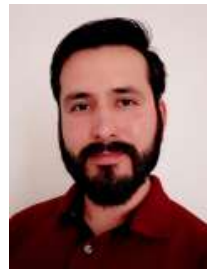

Tonatiuh Hernández received the B.Sc. in Computer Science and M.Sc. degree in Electrical Engineering and Computer Science from the University of Guadalajara, Mexico, in 2012 and 2016, respectively. Currently he is pursuing a Ph.D. in Electrical and Computer Science at the University of Guadalajara. He is an assistant professor in the Department of Computer Science at the University of Guadalajara, Mexico. His research interests are nonlinear and adaptive control, teleoperation of mobile manipulators and multiagent systems coordination.

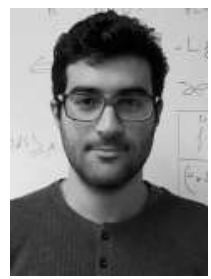

Mohamed Maghenem received his ControlEngineer degree from the Polytechnical School of Algiers, Algeria, in 2013, his M.S and Ph.D. degrees in Control from the University of Paris-Saclay, France, in 2014 and 2017, respectively. He is currently a Postdoctoral Fellow at the Electrical and Computer Engineering Department at the University of California at Santa Cruz. His research interests include dynamical systems theory (stability, safety, reachability, robustness, and synchronization), control systems theory (adaptive, time-varying, linear, non-linear, hybrid, robust, etc.) with applications to power systems, mechanical systems, and cyberphysical systems.

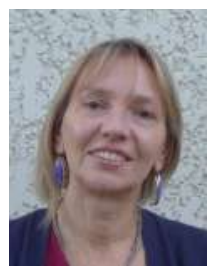

Elena Panteley received the M.Sc. and Ph.D. degrees in applied mathematics from the State University of St. Petersburg, St. Petersburg, Russia, in 1986 and 1997, respectively. From 1986 to 1998 , she held a research position with the Institute for Problem of Mechanical Engineering, Russian Academy of Science, St. Petersburg. Since 2004 she holds a tenure position as Senior Researcher of the French National Centre of Scientific Research (CNRS), at the Laboratoire de signaux et systmes, France. She is also associate researcher of ITMO University, St Petersburg Russia, since 2014. Her research interests include stability and control of nonlinear dynamical systems, network systems with applications to electromechanical, neuronal and more recently, social systems. 